\title{
High magnification crack-tip field characterisation under biaxial conditions
}

\author{
B. Moreno, P. Lopez-Crespo, J. Zapatero \\ Department of Civil and Materials Engineering, University of Malaga, Malaga (Spain)
}

\begin{abstract}
This work presents a novel methodology for characterising fatigue cracks under biaxial conditions. The methodology uses high magnification Digital Image Correlation (DIC) technique for measuring displacement and strain crack-tip fields. By applying micro-speckle pattern on the metal surface it is possible to achieve high magnification for DIC technique. The speckles were created by electro-spray technique. The validity of this novel technique is demonstrated by direct comparison with standard extensometer measurements, under tension-compression and torsion conditions. In order to image the correct region, the notch effect on the fatigue life was also evaluated.
\end{abstract}

KEYWORDS. Biaxial fatigue; Digital image correlation; Crack-tip strain field; Electro-spray.

\section{INTRODUCTION}

$\mathrm{N}$ umerous engineering components and structures are subjected to biaxial loading conditions. In these cases two principal stresses and strains change in time, both in magnitude and directions. However, due to biaxial experimental tests being difficult, expensive and time-consuming, the number of experimental data available in the literature is much limited than that for uniaxial fatigue. The aim of this work is to develop a methodology capable of characterising crack-tip fields under biaxial conditions. A procedure to enforce the crack initiation at a specific location without changing the fatigue life is explained. Then a new method for measuring displacement and strain crack-tip fields on fatigue cracks grown following the previous procedure is shown. This method is based on high-magnification Digital Image Correlation (DIC) technique. Surface finish required by DIC was achieved by electro-spray technique.

\section{MATERIALS AND METHODS}

7 he material investigates was St-52-3N steel with the chemical composition shown in Tab. 1. This is a low carbon steel often employed in structural applications.

\begin{tabular}{ccccccccc}
\hline Alloy & $\mathrm{C}$ & $\mathrm{Si}$ & $\mathrm{Mn}$ & $\mathrm{P}$ & $\mathrm{S}$ & $\mathrm{Cr}$ & $\mathrm{Ni}$ & $\mathrm{Mo}$ \\
St-52-3N & 0.17 & 0.225 & 1.235 & 0.010 & 0.0006 & 0.072 & 0.058 & 0.16 \\
\hline
\end{tabular}

Table 1: Chemical composition in weight $\%$ of St-52-3N steel. The balance is Fe.

Tubular hollow specimens with the dimensions described in Fig. 1 (RHS) were used in this work. All experiments were conducted with MTS 809 servo-hydraulic loading rig, allowing applying tension-compression-torsion load. Biaxial 
extensometer Epsilon 3550 was employed for axial and angular strain measurement. The experimental setup employed in this work is shown in Fig. 1.
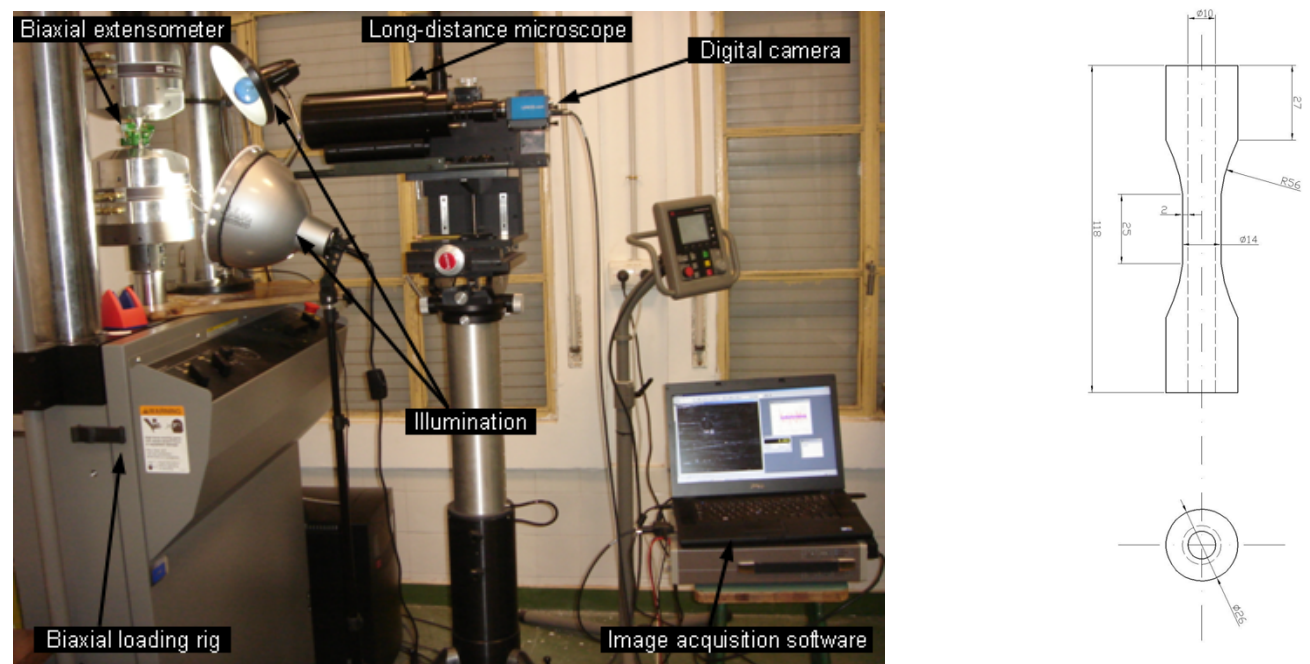

Figure 1: (LHS) Experimental setup and (RHS) geometry of the specimen employed in the experiments. Dimensions are in mm.

\section{METHODOLOGY TO STUDY CRACK INITIATION}

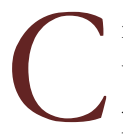

rack initiation stage was studied under combined tension-compression and torsion tests. Strain control mode was used during the experiments with cyclic sinus signal where the mean strain was zero $(\mathrm{R}=-1)$. At this stage proportional loading with amplitudes $\varepsilon_{\mathrm{a}}=0.0015$ and $\gamma_{\mathrm{a}}=0.0032$ was applied. The objective is to measure the initiation and growth of cracks with size ranging from a few hundreds microns up to $2 \mathrm{~mm}$. This was done by means of a long distance microscope capable of imaging field of view of $2 \times 1.5 \mathrm{~mm}^{2}$. However, it is very complicated to know a priori the exact spot where the crack will nucleate, given the cylindrical shape of the specimens. Moreover, current biaxial extensometers hide a large portion of the sample, making it extremely difficult to find the nucleation point with the long distance microscope. Therefore the geometry of the specimen needs to be modified so as to force the initiation of the crack at a particular spot and focus the microscope right there.

Different possibilities were explored. The first option was to use cylindrical specimens where the thickness was minimum at the central section, similarly to hour glass specimens [1]. However this option would not solve the problem, since although the crack would initiate at a particular section (that of minimum thickness), it could appear anywhere around the circumference. Moreover this geometry would condition the crack to grow in specific orientations. This could be avoided by introducing a very small stress concentrator. By making the concentrator of circular shape, it will not condition the direction of initiation of the crack. Thus, the second option investigated was to insert an indentation with a hardness testing facility. The main advantages of this option are the experimental simplicity and the repeatability that can be achieved by employing the same load and the same penetrator. Three indentations were introduced in a sample with a conical penetrator. The diameter of the mark left by the penetrator was smaller than $200 \mu \mathrm{m}$. However, it was observed that after applying 40000 cycles not only the specimen did not fail from any of these indentations, but also no single crack nucleated around the indentations. This is explained by the fact that the material work hardens around the indentation, thus being more resistant to fatigue growth in these spots. The third option explored consisted of drilling a very small hole. The hole should be large enough to force the crack nucleation around it, but small enough to induce a damage equivalent to that of the defects existing already in the material. The effect of holes of different diameter was thus analysed. The fatigue life of different samples with holes of diameters 1.25, 1, 0.6, 0.2 and $0.15 \mathrm{~mm}$ and no hole were studied, following Murakami's ideas [2]. The depth of the hole in all cases was approximately the same dimension as the diameter. The results are shown in Fig. 1. All the experiments were stopped when the axial load or torque dropped $20 \%$. The crack length at the end of each experiment was approximately $20 \mathrm{~mm}$. A crack nucleated around the hole in the sample with $0.15 \mathrm{~mm}$ drilled hole and fatigue life of 43500 cycles. However that crack did not propagate and instead the dominant crack did not originate at the hole. The results from Fig. 1 show that the fatigue life is reduced as the size of the hole is increased. For the largest hole tested, the fatigue life was reduced by $90 \%$ approximately. The fatigue life increases 
approximately in a linear way as the size of the hole is reduced, up to a size of approximately $0.15 \mathrm{~mm}$. By reducing further the size of the hole below $0.15 \mathrm{~mm}$ no change in the fatigue life is observed. A similar behaviour was previously found on other steels [2]. Hence holes of size $0.15 \mathrm{~mm}$ and smaller would not be expected to be detrimental to fatigue life. This is also supported by the fact that one of the samples with $0.15 \mathrm{~mm}$ hole did not fail at the hole and instead it failed from a crack nucleated elsewhere. Accordingly, drilling a hole of $0.15 \mathrm{~mm}$ diameter was chosen to force the crack to nucleate at a particular spot without affecting the fatigue properties of the material.

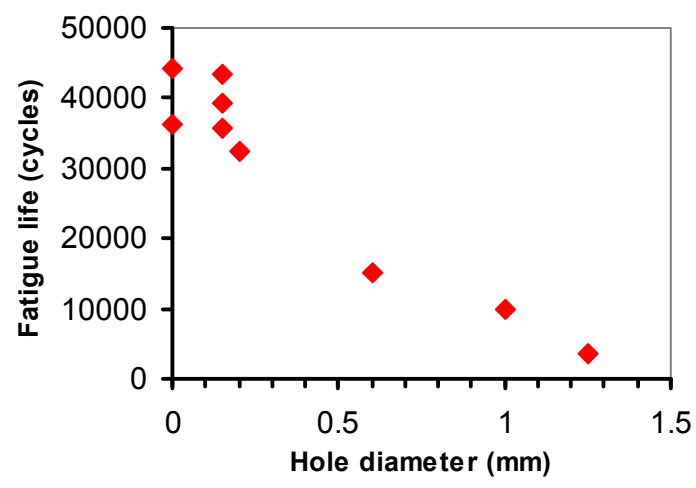

Figure 2: Fatigue lives for the different sizes of holes drilled investigated.

\section{DIGITAL IMAGE CORRELATION TECHNIQUE}

$\mathrm{D}$ igital Image Correlation (DIC) allows experimental measurement of displacement and strain fields in mechanical components. Important progress in the last decade in the field of digital photography gave rise to a more robust and accurate technique, in particular in the fields of fatigue and fracture [3, 4]. DIC equipment is relative simple and consists of a digital camera, a computer and specific software to process the images. The technique works by comparing digital images with different strain levels. Each image is subdivided in interrogation windows or subsets, so that the change in position of each of these interrogation windows is identified by comparison with a reference image. The displacement of each interrogation window is determined by minimising a cross-correlation function. Accurate results can be achieved by imaging non-uniform surfaces, so that each interrogation window can be identified uniquely in the deformed image. This non-uniform finish is normally obtained by slightly spray painting the surface with black and white [3-6], although other alternatives have also been developed [7].

In this work DIC technique has been employed to measure experimentally displacement and strain fields in cylindrical hollow specimens such as those described in the previous section, subjected to tension-torsion loads. DIC commercial package Vic-2D was employed for data processing. Images were captured with a long-distance microscope (Questar QM100) and a 5 Mega-pixel digital camera with 8 bit dynamic range. Due to the high level of magnification achieved with the microscope, the speckle pattern has to be extremely fine. This cannot be achieved by conventional spray-painting. It is thus the aim of this work to develop a technique capable of producing a pattern with ultra-fine speckles. The possibility of electro-hydrodynamic atomisation by means of electro-spray technique will be studied for this purpose. First, the methodology for generating the micro-speckle pattern is presented. The validity of the methodology is then studied by comparison with standard extensometer measurements. Finally the new procedure is used to characterise crack-tip displacement and strain fields under biaxial conditions (tension and/or torsion).

\section{MICRO-SPECKLE GENERATION BY MEANS OF ELECTRO-SPRAY TECHNIQUE}

$\mathrm{E}$ lectro-spray technique consists of extruding a liquid through a needle subjected to an electric field. By introducing solid particles in the liquid, the very fine jet coming out of the needle produces very small drops which get attached to the surface. Once the liquid is evaporated, the drops will give rise to micro or nanospeckles [8].

The most common electro-spray setup consists of a capillary with sharp ending through which a pumped liquid flows. A high voltage is then applied between the sharp edge of the capillary and a collector electrode. The collector electrode can have either zero voltage or the negative voltage of the capillary (see Fig. 3 LHS). 

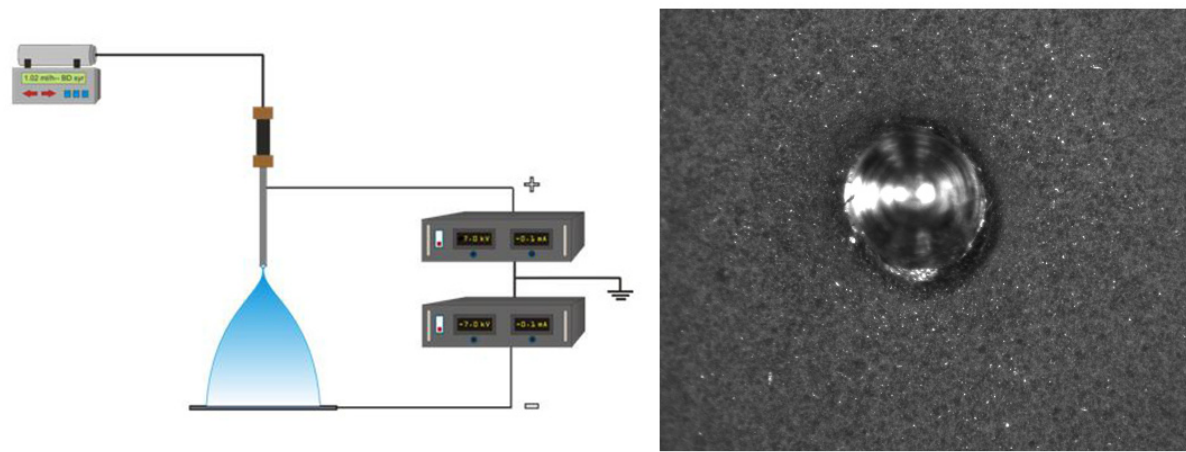

Figure 3: (LHS) Schematic of the setup used for electro-spray technique. (RHS) Speckle pattern produced by electro-spray. The diameter of the centre hole is $0.5 \mathrm{~mm}$.

The free charges of the liquid transform the shape of the liquid located at the ending of the capillary from spherical shape to conical shape. The thereby created cone is called Taylor cone [8]. The high charge of the drops induces repulsion forces between them. The smaller drops appear in the outer area of the cone and the heavier and larger drops tend to fall in the central axis of the spray [9]. There are two main parameters governing the electro-spray process: (i) the electric current flowing through the cone and (ii) the size of the spray drops. The diameter of the drops ranges between dozens of nanometres to hundreds of microns. Both parameters can be controlled by the flow rate and the properties of the fluid, namely electric conductivity, surface tension, viscosity, density and electric permittivity [10, 11]. To a first approximation, the fluid conductivity allows controlling the order of magnitude of the drop diameter. The flow rate allows a smaller range once the conductivity is fixed.

The size of the drops should be small enough so that each of the interrogation windows in which the image is divided can be indentified uniquely by the cross-correlation algorithm. Drop sizes of $3 \times 3$ pixels are typically recommended to obtain good results. For the magnification level employed with the current long-distance microscope, the resolution is approximately $1 \mu \mathrm{m} /$ pixel. Accordingly, the size of the drops should be around $3 \mu \mathrm{m}$.

Black and white inks were chosen for better contrast. The black ink of was made of dimethylformamide (DMF), Vulcan carbon with xylene and polyethylene vinyl acetate (PEVA). The addition of PEVA improved the adhesiveness of the ink to the metal surface. This adhesiveness is much required since once the speckle is applied onto the surface, the paint can be detached during the handling of the specimen. This includes the drilling of the hole, placing the specimen in the grips of the loading rig and placing the extensometer in the sample. In order to increase the conductivity of the solution, antistatic agent Stadis was also added. This was the black solution that was used as background on the metal surface. White speckles were created with a solution made of methylene chloride and methanol with titanium oxide $\left(\mathrm{TiO}_{2}\right)$. The solvent was chosen so that flow rates between 0.04 and $2 \mathrm{ml} /$ hour and voltage of $60 \mathrm{kV}$ can be used. The flow rate and voltage values were given by the pump and the voltage source respectively. An example of the speckle pattern generated is shown in Fig. 3 RHS.

\section{STRAIN RESULTS IN UN-CRACKED SPECIMENS}

$\mathrm{I}$ $\mathrm{n}$ order to validate the experimental setup described in the previous sections, DIC strain data were compared with strain information measured with a biaxial extensometer. Cylindrical shaped hollow un-cracked specimens were used. These were subjected firstly to uniaxial tension-compression cyclic load and secondly to biaxial tensioncompression-torsion in-phase cyclic load. Tests were made with load and torque control mode on the loading rig at a 0.2 $\mathrm{Hz}$ frequency. This frequency allowed the acquisition of 65 images by the digital camera, providing enough information to describe a cycle. The reference image for DIC was the one giving zero strain with the extensometer. Since no stress concentrator was introduced in these specimens, the displacements both in the axial and the angular direction were linearly proportional to the distance from the grips. Therefore, uniform distribution in axial and angular strain fields is expected in the calibrated length of the specimen. Fig. 4 LHS shows a comparison of the axial strain measured by both DIC technique and extensometer. These were obtained under tension-compression uniaxial test with loads ranging between $\pm 15 \mathrm{kN}$. Fig. 4 RHS shows a comparison of the angular strain measured by both DIC technique and extensometer. Tension-compression-torsion loads were applied during the test shown in Fig. 4 RHS, with axial loads being $\pm 15 \mathrm{kN}$ and torsion torque $\pm 70 \mathrm{Nm}$. Similar agreement between DIC and extensometer were obtained in biaxial 
test in both axial and torsion components. The very good agreement observed in Fig. 4 between DIC and extensometer data demonstrate the validity of the experimental proposed method.

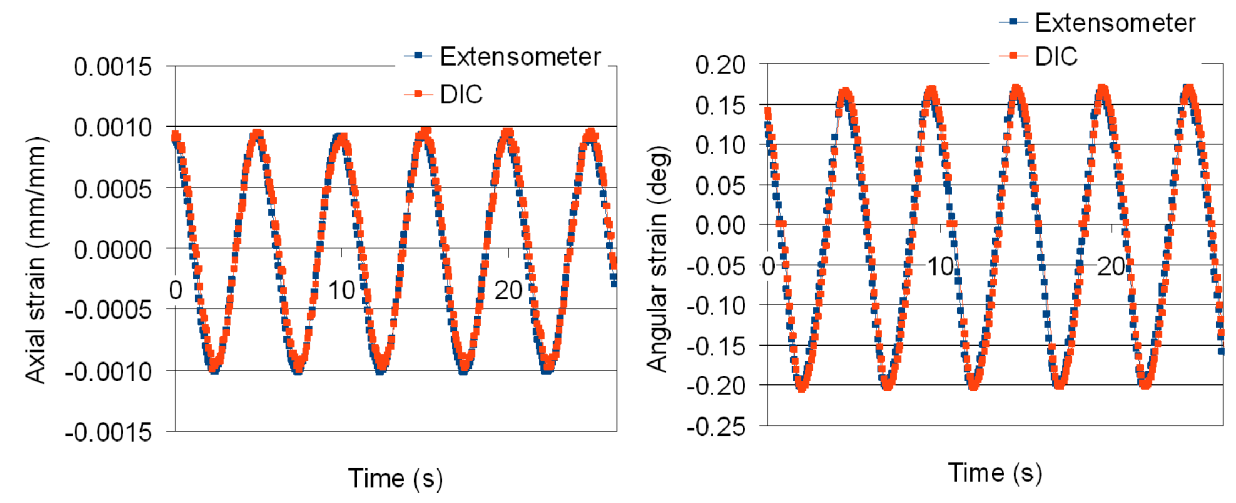

Figure 4: (LHS) Axial strain and (RHS) angular strain evolutions along 5 cycles measured by DIC and biaxial extensometer.

\section{STRAIN RESULTS IN CRACKED SPECIMENS}

$\mathrm{U}$ $\mathrm{n}$-cracked specimens were used to validate the methodology combining DIC and long-distance microscope on biaxial samples. Once the methodology was validated, it is applied to study the crack-tip field around fatigue cracks. A $0.15 \mathrm{~mm}$ diameter hole was drilled in a sample. The sample was then subjected to $\pm 15 \mathrm{kN}$ axial load and $\pm 70 \mathrm{Nm}$ torsion torque at a $3 \mathrm{~Hz}$ frequency. Axial load and torsion torque were in-phase. However, due to the small stress concentrator effect of the $0.15 \mathrm{~mm}$ diameter hole, the crack was initiated in a region far from the hole and thus it could not be detected nor measured with DIC. It was then decided to drill a larger hole $(0.5 \mathrm{~mm}$ diameter $)$ to enforce the crack initiation from the hole (see Fig. 3 RHS). Every 2500 cycles, the frequency was decreased from 3 to 0.2 $\mathrm{Hz}$ so that each cycle could be studied with sufficient number of images (65 images per cycle). 5 complete cycles were captured by acquiring images for 30 seconds. Subsequently, the frequently was set to $3 \mathrm{~Hz}$ again and 2500 cycles were applied before the following measurement.

Fig. 5 shows the vertical displacement field, as well as a displacement profile along a vertical line after 100 cycles were applied on the specimen. No crack was detected in Fig. 5. A linear displacement distribution along the vertical direction is also observed in Fig. 5. Very similar results were obtained in un-drilled samples, thus suggesting the little stress concentrator effect of such hole. Figs. 6 LHS and RHS show the strain field after applying 27500 and 30000 cycles respectively. The stress concentrator effect can be easily appreciated in Figs. 6 in two diametrically opposite regions around the hole. The growth direction of the crack can be estimated from Fig. 6 to be approximately $30^{\circ}$ with respect the longitudinal (vertical) axis of the specimen. By carefully examining the hole, the crack can be only appreciated on the top right area and bottom left area of the hole. However, it is not possible to distinguish the crack in the speckle-painted surface. Nevertheless, the strain maps in Figs. 6 clearly show evidence of a crack.
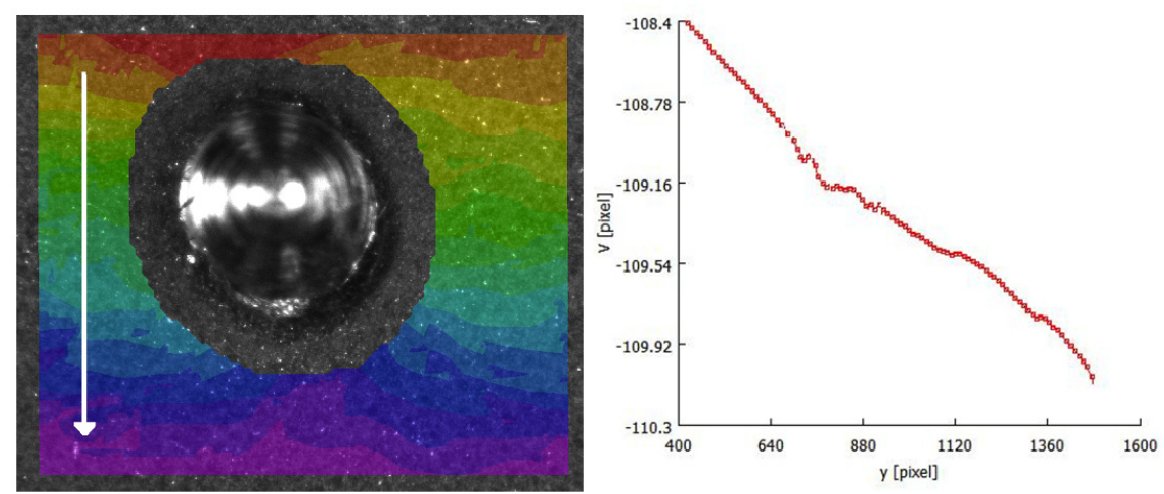

Figure 5: (LHS) Vertical displacement field and (RHS) displacement profile along a vertical line (shown in white) after applying 100 cycles on the specimen. 

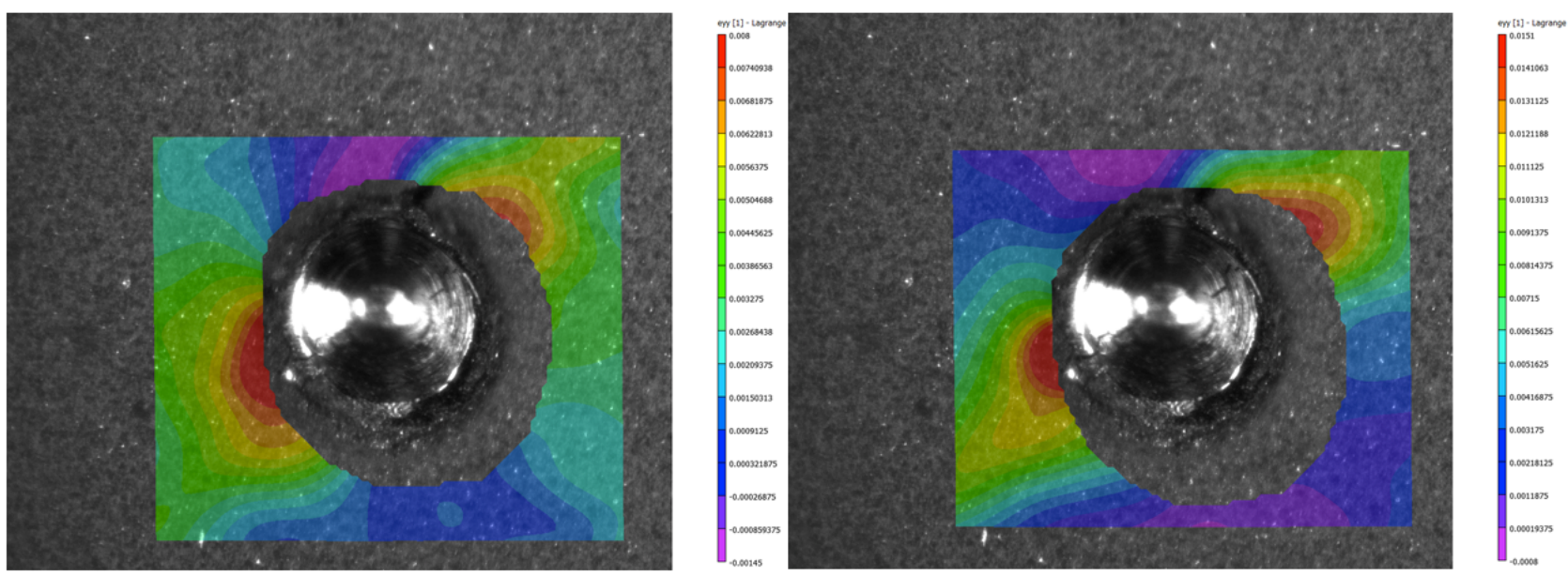

Figure 6: Strain strain field after applying (LHS) 27500 and (RHS) 30000 cycles.

Fig. 7 shows the vertical displacement map as well as a vertical displacement profile along crack opening direction. Fig. 7 was obtained by rotating the camera some $60^{\circ}$ clockwise so that the crack appears horizontal in the image. A drastic change due to the existence of a discontinuity is observed in the displacement profile (RHS in Fig. 7). Therefore, after applying 33500 cycles (Fig. 7) it is possible to distinguish the crack. At this stage, the crack can be distinguished as a discontinuity in the vertical displacement field (RHS in Fig. 7). The crack is also visible by looking at the painted surface because of the paint being cracked around the fatigue crack mouth. Rotation of the camera also allows easier identification of the crack as a mode I (opening mode) crack. Due to the discontinuity introduced by the crack, the algorithm is not able to provide displacement information just above and below the crack plane, since displacements in these positions are very different. This behaviour is most probably due to the fact that the crack in the metal is longer than that in the paint. When the crack is very short (up to approximately 30000 cycles in the current specimen), the paint is elastically deformed without being cracked. For longer crack lengths (from 33500 cycles onwards) the crack opening displacement in the metal is so large that elastic deformation of the paint is not enough to accommodate such large displacement. This causes the paint to break around the region close to the metal crack mouth. The fact that the crack length shown by the paint is smaller than the real crack length in the metal is studied in Fig. 8 . Fig. 8 shows a superposition of the metal surface after being cleaned (i.e. with no paint) and vertical strain fields calculated by DIC. Comparison between Figs. 7 and 8 makes clear that actual crack length in the metal (approximately $3.2 \mathrm{~mm}$ ) is much longer than that in the paint (approximately $0.86 \mathrm{~mm}$ ). Therefore, with the current arrangement, it is not possible to directly measure the correct crack length in the speckled surface.
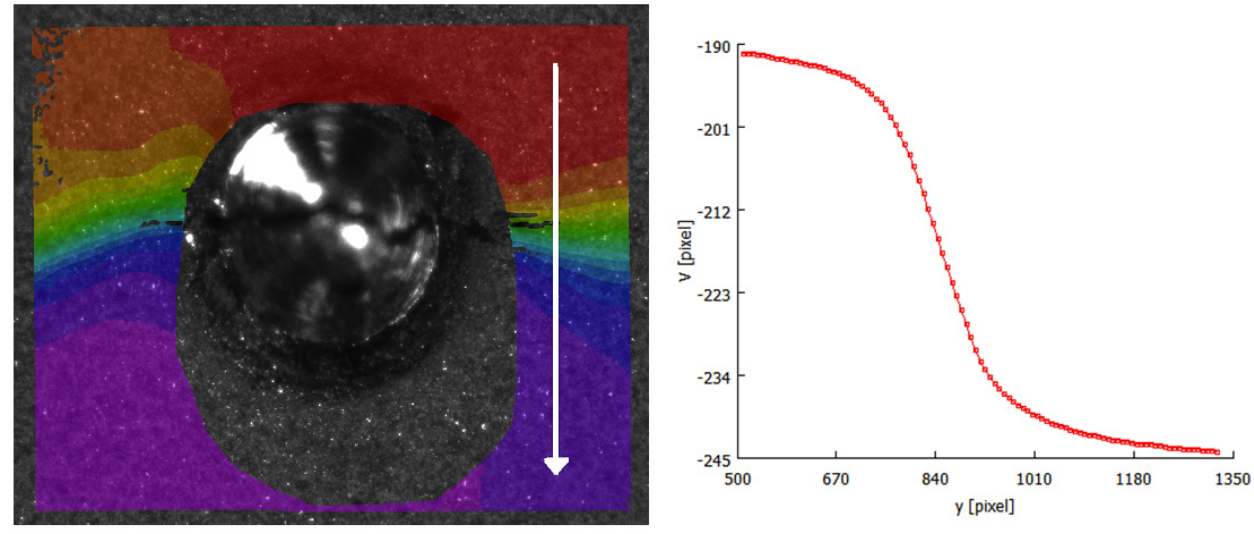

Figure 7: (LHS) Vertical displacement field and (RHS) displacement profile along a vertical line (shown in white) after applying 33500 cycles on the specimen 


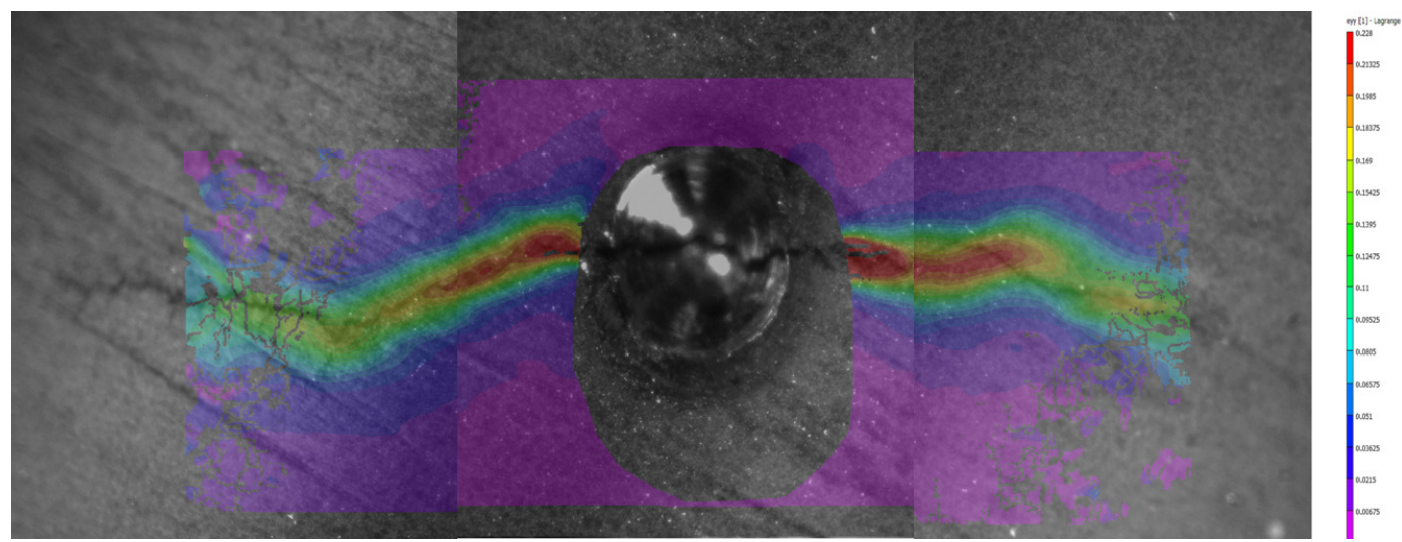

Figure 8: Superposition of the metal surface after being cleaned (i.e. with no paint) and vertical strain fields calculated by DIC after applying 33500 cycles.

\section{CONCLUDING REMARKS}

$\mathrm{F}^{\mathrm{n}}$

rom the results obtained, the following conclusions can be drawn:

A new procedure based on Murakami's ideas for enforcing the crack initiation at a specific location was successfully developed.

Electro-spray technique was effectively employed for producing a micro-speckle pattern that can be used by DIC technique combined with long-distance microscope to evaluate crack-tip displacement and strain fields.

- Electro-spray technique for creating the speckle pattern required by DIC did not allow the monitoring of the crack length, since it obscures the crack. Better results could be obtained when monitoring the crack length by making the speckle paint film not continuous. However this would also imply a lower contrast and so further experiments are required to find a solution.

\section{ACKNOWLEDGEMENTS}

$\mathrm{F}$ inancial support of Spanish Ministerio de Economía y Competitividad through grant reference DPI2012-33382 is greatly acknowledged. Experimental help by Mr F Sabarit is also much appreciated.

\section{REFERENCES}

[1] ASTM E606 / E606M - 12 Standard test method for strain-controlled fatigue testing. (2012).

[2] Murakami, Y., Metal Fatigue: effects of small defects and non metallic inclusions Oxford, UK: Elsevier, (2002) 35-56.

[3] Sutton, M. A., McNeill, S. R., Helm, J. D., Chao, Y. J., Advances in two-dimensional and three-dimensional computer vision. In: Photomechanics, Topics in Applied Physics, Rastogi PK, editor., 77 (2000) 323-372.

[4] Fonseca, J. Qd., Mummery,P. M. , Withers, P. J., Full-field strain mapping by optical correlation of micrographs acquired during deformation, Journal of Microscopy, 218(1) (2005) 9-21.

[5] Yoneyama, S., Ogawa, T., Kobayashi, Y., Evaluating mixed-mode stress intensity factors from full-field displacement fields obtained by optical methods, Engineering Fracture Mechanics, 74 (2007) 1399-1412.

[6] Roux, S., Hild, F., Stress intensity factor measurements from digital image correlation: post-processing and integrated approaches, International Journal of Fracture, 140 (2006) 141-157.

[7] Lopez-Crespo, P., Shterenlikht, A., Patterson, E. A., Withers, P. J., Yates, J. R., The stress intensity of mixed mode cracks determined by digital image correlation, Journal of Strain Analysis for Engineering Design, (2008) 43.

[8] Taylor, G. I., Disintegration of water drops in an electric field. Proceedings of the Royal Society - A, 280 (1964) 383397.

[9] Deng, W., Gomez, A., Influence of space charge on the scale-up of multiplexed electrosprays, Journal of Aerosol Science, 38 (2007) 1062-1078. 
[10] Mora, J. Fdl., Loscertales, I. GJ., The current emitted by highly conducting Taylor cones, Fluid Mechanics, 260 (1994) 155-158.

[11] Gañán-Calvo, A., Dávila, J., Barrero, A., Current and droplet size in the electrospraying of liquids Scaling Laws, Journal of Aerosol Science, 2 (1997) 249-275. 